Research Article

\title{
Scalable Deposition of Nanomaterial-Based Temperature Sensors for Transparent and Pervasive Electronics
}

\author{
Andreas Albrecht $\mathbb{D}^{1},{ }^{1}$ Almudena Rivadeneyra $\mathbb{D}^{1},{ }^{1}$ Marco Bobinger $\mathbb{D}^{\mathbb{D}}{ }^{1}$ \\ Jacopo Bonaccini Calia, ${ }^{1}$ Florin C. Loghin, ${ }^{1}$ Jose F. Salmeron (iD, ${ }^{1}$ Markus Becherer, \\ Paolo Lugli, ${ }^{2}$ and Aniello Falco ${ }^{2}$ \\ ${ }^{1}$ Institute for Nanoelectronics, Technical University of Munich, Munich, Germany \\ ${ }^{2}$ Faculty of Science and Technology, Free University of Bolzano, 39100 Bolzano-Bozen, Italy \\ Correspondence should be addressed to Almudena Rivadeneyra; arivadeneyra@ugr.es
}

Received 11 April 2018; Revised 8 August 2018; Accepted 16 August 2018; Published 31 October 2018

Academic Editor: Sang Sub Kim

Copyright (c) 2018 Andreas Albrecht et al. This is an open access article distributed under the Creative Commons Attribution License, which permits unrestricted use, distribution, and reproduction in any medium, provided the original work is properly cited.

This work presents a comparative analysis of materials for planar semitransparent thermocouples fabricated by spray deposition on a flexible substrate. Three different materials are employed to build such devices, analyzing also the effect of the spray order in their final performance. The highest Seebeck coefficient $(50.4 \mu \mathrm{V} / \mathrm{K})$ is found for a junction made of carbon nanotubes (CNTs) on top of silver nanowires (AgNWs) whereas its efficiency in terms of power is the lowest because of the higher sheet resistance of the CNTs. In this case, the best combination for energy-harvesting purposes would be poly (3,4-ethylenedioxythiophene) polystyrene sulfonate (PEDOT:PSS) and AgNWs, with a power factor of $219 \mathrm{fW} / \mathrm{K}^{2}$. These results prove the feasibility of developing large-scale and cost-effective thermocouples that could be used for sensing or energy-harvesting applications.

\section{Introduction}

Thermoelectric (TE) phenomena have been investigated and employed extensively for more than a century, as they relate two fundamental quantities: electricity and thermal transmission. Among the many TE effects which have been studied and exploited, the two which received a broader attention are the Peltier and Seebeck effects, respectively. These two can be seen as counterparts, since the former explains a temperature gradient due to electrical current flow through dissimilar materials while Seebeck is mainly the potential difference due to a temperature gradient [1].

The latter can be employed for the realization of "thermocouples," ideally defined as the series of two lines of different materials, which generate voltages with opposite signs upon the application of a thermal gradient. By applying a temperature gradient between the junction of the two materials and their extremities, it is possible to measure a potential difference between the single ends, which will be the sum of the voltages generated by each "leg" [2]. A first order mathematical expression of the Seebeck effect for each line is the following:

$$
\Delta V=\alpha_{S} \Delta T
$$

with $\alpha_{S}$ being the material-dependent Seebeck coefficient sometimes also referred to as simple "S."

Since (1) shows a linear dependency between temperature change and resulting voltage, this effect is a wellsuited indicator for temperature measurement or energyharvesting applications. For many material combinations, this relationship is linear, leading to an easy-to-read temperature sensor. In addition to this, a steep curve is preferred, as it represents a thermocouple more sensitive to temperature changes and allows a higher possible resolution. Remarkably, the insurgence of a voltage difference can also be employed to drive an electric load or to charge up an energy accumulator. The usage of TCs for thermal energy-harvesting applications has been driven by materials with very high thermoelectric power, such as bismuth 
telluride $\left(\mathrm{Bi}_{2} \mathrm{Te}_{3}\right)$ [3]. The ability to convert thermal energy in electricity can be effectively summarized by the dimensionless figure of merit (FoM) ZT, defined as

$$
\mathrm{ZT}=\alpha_{S}^{2} \sigma \frac{T}{K}
$$

where $\sigma$ is the electrical conductivity and $K$ is the thermal conductivity [4].

Together with $\alpha_{S}$, these transport parameters are intertwined and are affected by other intrinsic properties of the material, such as carrier concentration and band structure [4]. The FoM also gives clear indications of how more efficient thermocouples can be obtained by either maximizing $\alpha_{S}$ and $\sigma$ or minimizing $K$.

Nevertheless, these strictly thermoelectrical parameters alone do not suffice to define next generation thermocouples. In fact, the research in the field of the Internet of Things and wearable electronics has been leaning towards the realization of flexible or conformal devices [5], seamlessly integrated in the environment [6], objects [7], or even the human body [8]. Furthermore, conventional thermocouples are built of different metals, metal alloys, or doped semiconductors, which present high ZT values as well as high environmental impact. The deposition of pure metals and inorganic semiconductors is extremely energy intensive [9] and difficult to recycle. One approach towards the substitution of such materials is the realization of novel devices, based on polymers or nanostructured materials. Albeit, at the current state, nanomaterials present lower FoM; they still have a wide margin of improvement, and they possess a number of uncommon qualities, which are not to be found in conventional thermoelectric materials. Nanomaterials are potentially low cost [10], can be obtained through nontoxic production processes [9], can be semitransparent [11], and can be easily processed into various shapes $[12,13]$ on arbitrary substrates $[14,15]$.

For instance, it is possible to increase the $\mathrm{ZT}$ values of insulating or semiconducting polymers, through the simple addition of one-dimensional fiber structures, such as carbon nanotubes (CNTs) or conducting nanowires. A composite of polymer and fiber would preserve the low thermal conductivity of a polymer, while it would present an enhanced conductivity, due to the low electrical resistivity of the fiber structure, after reaching a percolation threshold [16]. An interesting example for this class of solutions is the work of $\mathrm{Yu}$ et al., in which they demonstrated a great increase of the FoM through adding CNTs into polymer [17].

In a similar direction, the employment of conductive nanomaterials (e.g., silver nanowires (AgNWs) [18], poly (3,4-ethylenedioxythiophene) polystyrene sulfonate (PEDOT:PSS) [19], and CNTs [20]) can improve the performance of the TCs because of very low heat capacity accompanied by Seebeck coefficients of circa $40 \mu \mathrm{V} /{ }^{\circ} \mathrm{C}[21,22]$. As previously stated, the interest in this class of materials is also due to their potential (semi)transparency. Nanotubes, nanowires, and polymers have been previously employed in many applications, which require a semitransparent conductive layer, such as organic photodiodes [23, 24], solar cells [25], organic light-emitting diodes [26], and transparent heaters [27] as well as power-harvesting applications [28-30].

In this work, we exploit all the remarkable properties of solution processable nanomaterials such as scalability and roll-to-roll (R2R) processability at ambient conditions to fabricate novel kinds of semitransparent thermocouples by means of spray deposition. Because of the inherit flexibility of this deposition technique, the novel devices we propose can be integrated straightforwardly and seamlessly onto any transparent surface, enabling the fabrication of semitransparent differential temperature sensors for smart homes and integrated cars or even for the smart lenses of the future.

\section{Materials and Methods}

2.1. Materials. For the fabrication of the TC, we tested different combinations of materials, in particular, a custom-made solution of PEDOT:PSS (Clevios PH1000) with $1 \mathrm{~g}$ of polymer diluted in $4 \mathrm{~g}$ of deionized (DI) water, $10 \mathrm{mg}$ of Dynol 604 (Sigma-Aldrich), and $250 \mathrm{mg}$ of Ethylene glycol (EG), which is employed to increase their conductivity [31]. Before adding PEDOT:PSS, the material was filtered with a pore-size filter of $0.45 \mu \mathrm{m}$. The solution was always sonicated for $30 \mathrm{~s}$ to disperse agglomerates before spray deposition. For AgNWs, we diluted $1 \mathrm{~g}$ of AgNW (ECD0X3from Ras Materials) with $14 \mathrm{~g}$ isopropyl alcohol and $5 \mathrm{~g}$ deionized (DI) water. The mixture thus produced, the solution was always shaken before using it.

The CNTs that we have used are commercially available from Sigma-Aldrich (Product number: 773735). The CNT ink consists of DI water, $90 \%$ semiconducting single-walled CNTs (SWCNTs), and sodium dodecyl sulfate (SDS, Sigma-Aldrich), which acts as dispersing agent. The base solution was made by dissolving 1 wt \% SDS in DI water. 0.03 wt \% of CNTs were added to the solution and treated with a horn sonicator (Branson Sonifier S-450D) for $25 \mathrm{~min}$ at a power of $50 \%$. The solution was then centrifuged at $15 \mathrm{krpm}$ for $90 \mathrm{~min}$ and the top $80 \%$ of the supernatant separated, to be used as the CNT ink [32].

2.2. Fabrication Process. To manufacture the TCs, the inks were sprayed through a shadow mask onto a $75 \mu \mathrm{m}$ thick Kapton HN foil with an area of $50 \times 50 \mathrm{~mm}^{2}$. We selected the polyimide film because of its high thermal stability to avoid influences of the substrate or its destruction during the measurement. In accordance with the process parameters that we previously tailored for the deposition of AgNWs and CNTs [27, 33], the materials were sprayed to conductive films using a fully automated spray robot PVA350 from Werner Wirth (Germany) equipped with a 781S General Purpose spray valve from Nordson (USA) [18]. Every layer was deposited via mesh-like motion, as shown in Figure 1. The nozzle movement had a speed of $250 \mathrm{~mm} / \mathrm{s}$, and the nozzleto-sample distance was set to $50 \mathrm{~mm}$; the mesh line distance was set to $3 \mathrm{~mm}$. All samples were sprayed with a material pressure of $0.02 \mathrm{MPa}$ and an atomizing pressure of $0.05 \mathrm{MPa}$. The orifice opening of the nozzle can be manually decreased with a valve (nozzle orifice opening value). The maximum diameter of the nozzle orifice is $0.3 \mathrm{~mm}$. The 


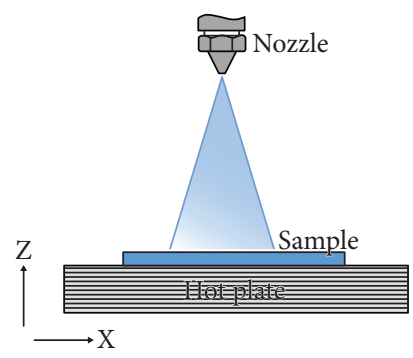

(a)

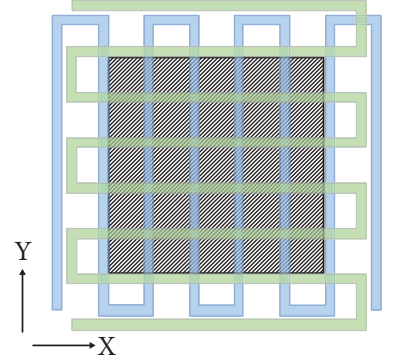

(b)

FIGURE 1: (a) Schematic of the spray setup. (b) Schematic of the trajectory of the gun over the sample while spraying: one deposited layer consists of spraying the sample in line form on $\mathrm{x}$ - and on $\mathrm{y}$-direction. The gun remains at the same distance of the sample during the whole process.

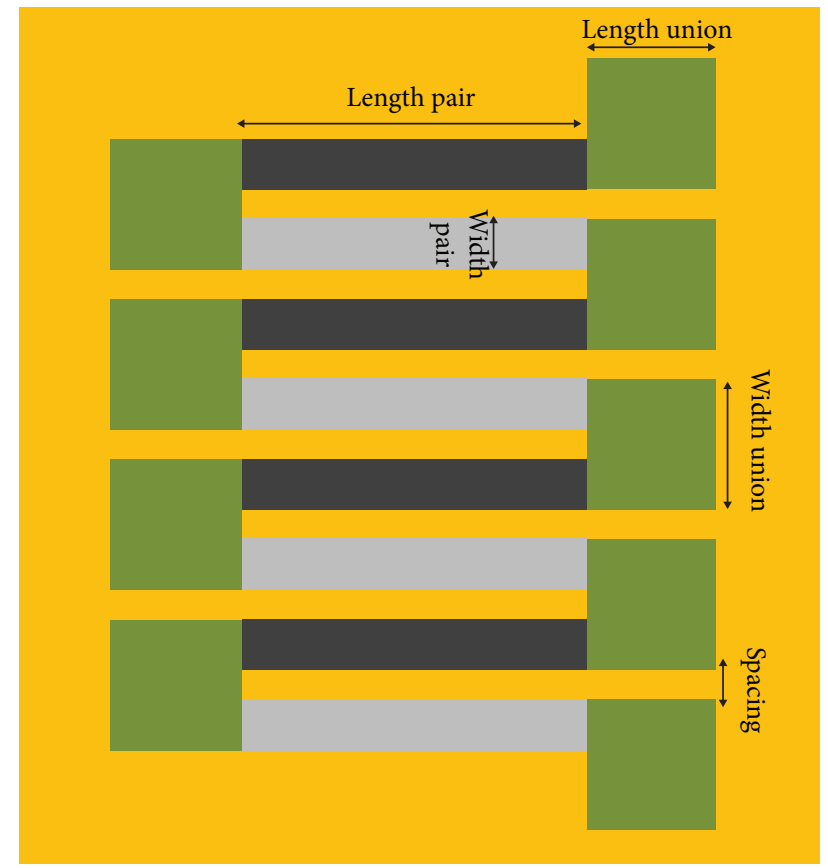

(a)

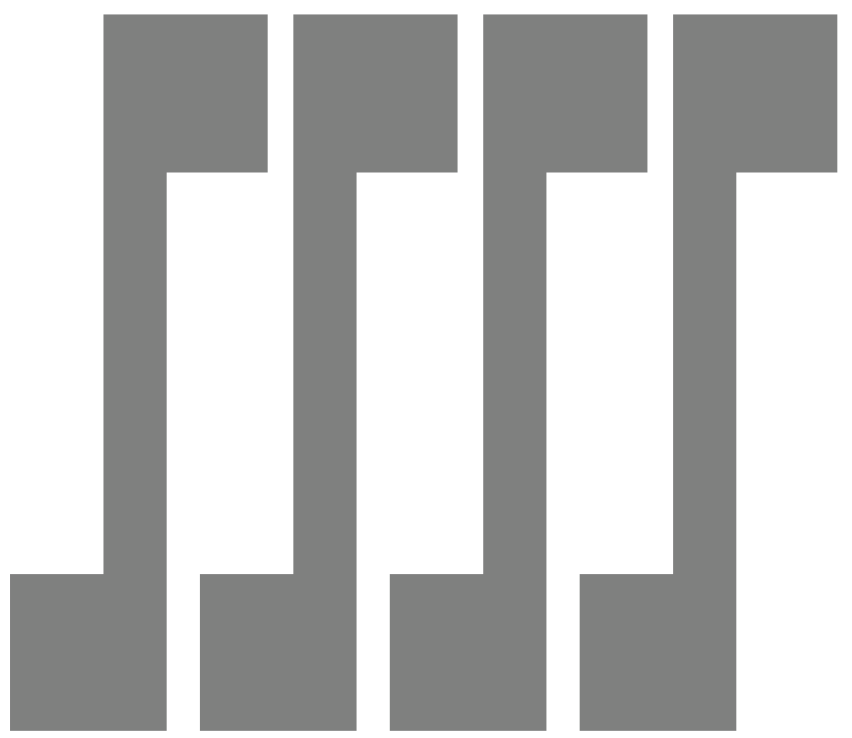

(b)

Figure 2: (a) Dimensions of one TC device containing four TC. The TC legs are all identical, $2.5 \mathrm{~mm}$ width and $27.5 \mathrm{~mm}$ length. The different colors in the drawing are indicating the different materials: in dark grey the material sprayed first, in light grey the material sprayed seconds, in green the contacts, which were made by the overlapping of both sprayed materials, and in yellowish color the substrate. (b) Layout of the lasercut mask needed to spray the four TC structures. Two such masks are needed to produce one TC. The first mask is oriented as in the picture. The second mask is the same, rotated by $180^{\circ}$, to be placed superimposing the mask ends in alternate manner. This creates the pattern shown in (a).

spraying nozzle was changed when spraying with CNT or AgNW/PEDOT:PSS, with its appropriate gun number, which is written manually on its metal frame. The temperature of the hot plate was also varied when spraying the different materials. In particular, the temperature was set to $65^{\circ} \mathrm{C}$ for AgNWs, $90^{\circ} \mathrm{C}$ for PEDOT:PSS, and $80^{\circ} \mathrm{C}$ for SWCNTs. The spray deposition of a solution implies to be in a wet spraying regime.

To speed up the drying of the wet droplets arriving at the substrate, the substrates were left lying on the hot plate. This approach allows the formation of dry layers with good thickness control over time, enhancing at the same time reproducibility [32].
Four TCs are fabricated in series (see Figure 2(a)), fixing the substrate and the shadow mask (see Figure 2(b)) via small magnets (diameter of $1 \mathrm{~mm}$ ) on the hot plate of the spray machine. Ten layers of each material were spray deposited, each having its own mask.

2.3. Characterization. The transmittance spectra were measured from $350 \mathrm{~nm}$ to $800 \mathrm{~nm}$ using the light from a $300 \mathrm{~W}$ Xenon arc lamp from Newport Oriel chopped at $210 \mathrm{~Hz}$, which passes through an Oriel Cornerstone 260 1/4 monochromator and illuminates a calibrated photodiode with a transconductance amplifier connected to an Oriel Merlin digital lock-in amplifier. 

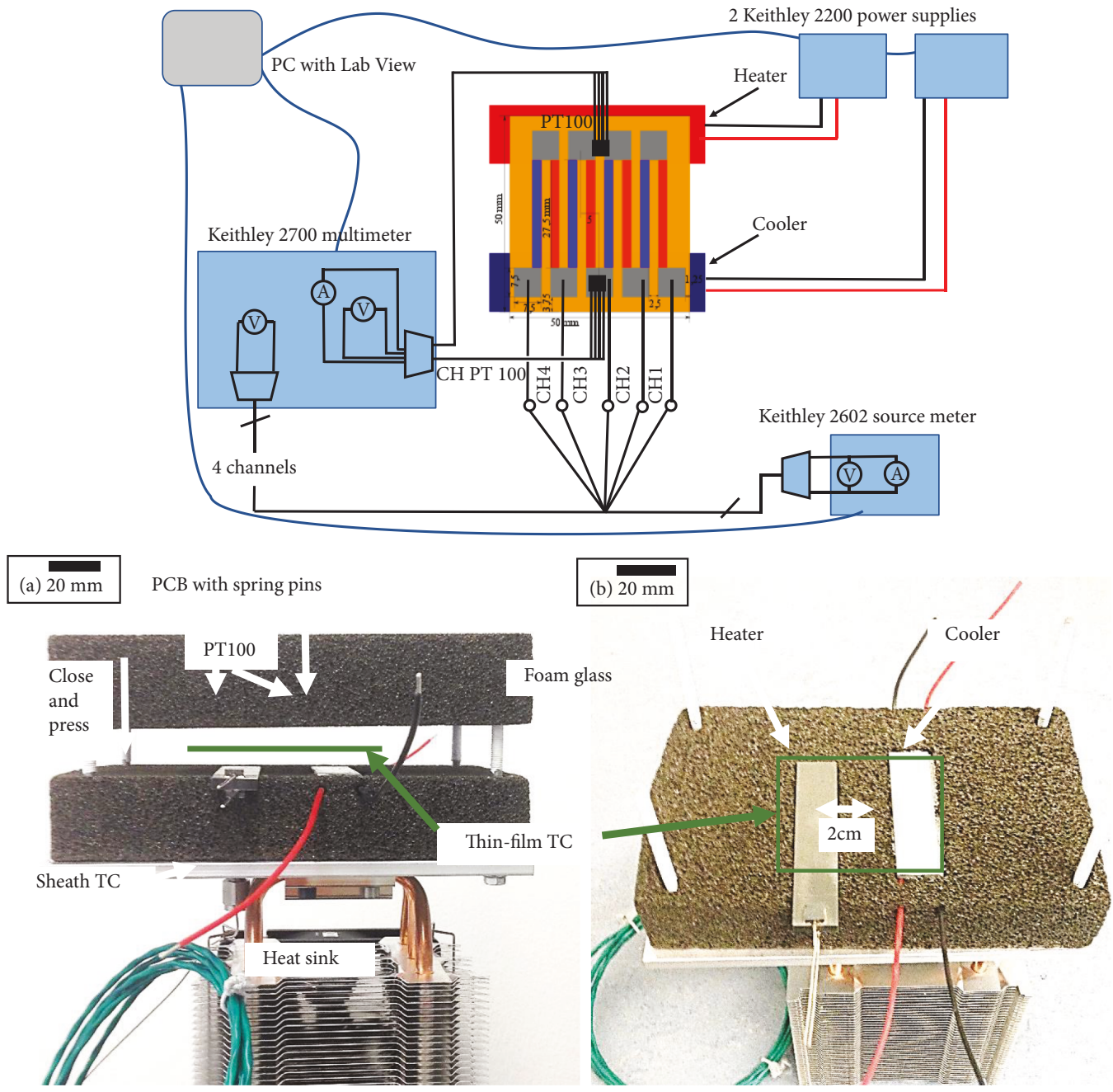

Figure 3: Custom-made setup for the thermoelectrical characterization of the device schematic on the top part, real picture on the bottom. The position of the TC under test is marked in green from (a) side view with lifted top cover and (b) top view without top cover. Reprocessed from original files published in [35].

To quantify the performance of a transparent conducting material, the ratio between the transmission at $550 \mathrm{~nm}\left(T^{10}\right)$ and the sheet resistance of the film $\left(R_{s}\right)$ is taken. The formula in (3) gives a FoM on conductive films [34].

$$
\mathrm{FoM}=\frac{T^{10}}{R_{S}} .
$$

Sheet resistance was measured at room temperature using a four-point probe head from Jandel connected to a Keysight B2901A source measuring unit.

In order to determine the exact thickness of the film, a tactile profilometer measurement is performed with the Bruker's DektakXT device.

A field-emission scanning electron microscope (FESEM) is used for the surface imaging. Therefore, it scans over the surface of the sample with an electron beam exposed to voltages between $1 \mathrm{kV}$ and $30 \mathrm{kV}$.

Infrared images were recorded using a high-resolution calibrated thermal imaging camera (Testo $890,640 \times 480$ pixels), which employs bolometric detectors and has a spectral sensitivity window of $7.5-14 \mu \mathrm{m}$. The emissivity of the thin films was determined by adjusting the temperature measured by the camera to match the temperature on the heated side, which was measured employing a PT100 thermoresistance.

A custom-built setup was designed to characterize the flexible transparent TCs. The setup consists of a flat ceramic heater and a Peltier cooler embedded into a low-heatconductive isolating foam glass. The setup is illustrated in Figure 3. The TCs are placed on top of the heater and cooler and pressed down by the top foam glass and a weight. The temperatures at the surface of the TCs are measured by thermoresistors (PT100 from Heraeus M422) mounted at the hot and cold side. Two single-channel programmable power supplies (Keithley 2200) control the heater and cooler. The voltages generated by the TCs are measured by a Keithley 2700 multimeter at an input impedance of $1 \mathrm{M} \Omega$. For all measurements, the temperature was ramped from $27^{\circ} \mathrm{C}$ to $107^{\circ} \mathrm{C}$, corresponding to a maximum temperature difference of $80^{\circ} \mathrm{C}$. The setup is automated by LabView 2016. 


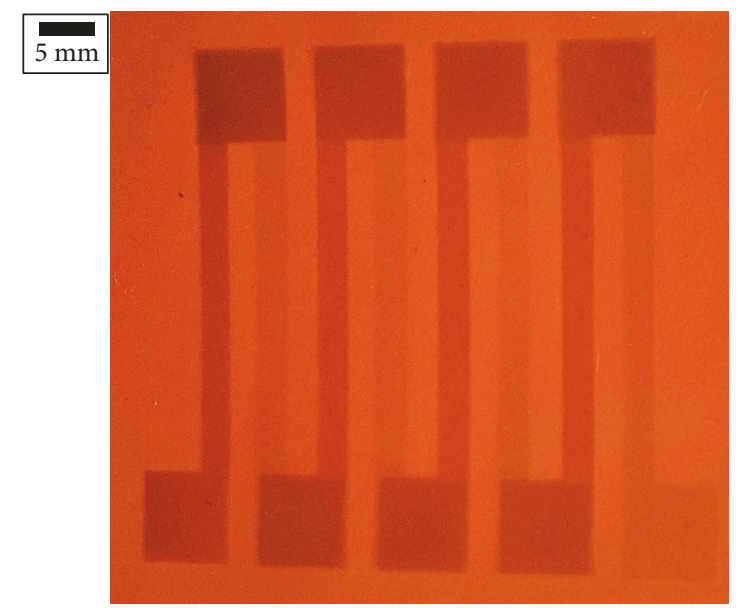

Figure 4: Picture of thermocouples made of PEDOT:PSS (dark grey) sprayed on top of AgNW (light grey). AgNWs form a milkywhite or silver layer and the blue/grey PEDOT:PSS layer overlaps at the contact regions. The hot area is on the right side and the cold area is on the left side similar to the schematic.

Infrared (IR) images for the thermocouple setup with the heater at a temperature of $300^{\circ} \mathrm{C}$ are included in the supporting information in Figure S1. Due to the excellent thermal insulation of the foam glass, the generated heat is confined to the heater area. This confinement gives rise to an increase in the temperature gradient and, hence, also an increase in the Seebeck coefficients.

\section{Results and Discussion}

3.1. Physical Characterization. Figure 4 presents a photograph of a manufactured series of four thermocouples made with PEDOT:PSS sprayed on top of AgNWs. The blueish color of PEDOT:PSS appears dark whereas the AgNW is less pronounced. The definition of the patterns is reproducible, and the error in the alignment is almost negligible (less than $1 \mathrm{~mm}$ in the worst case) in comparison with the junction area.

Thickness was measured against a reference level, the glass substrate itself, obtained by scratching with a thin scalpel a straight line on the film and using the scratch bottom as reference value for the zero-height value. The average thicknesses of the sprayed layers for all nanomaterials were below $300 \mathrm{~nm}$, corresponding to very little material usage. Thickness measurements are not well suited for nanowire and nanotube networks as they possess a low density and only peaks are visible. Scanning electron microscopy (SEM) is more suitable to evaluate the uniformity of the spray deposition. SEM was used to image different parts of the sample and verify the complete coverage of the sample. Figure 5 shows illustrative images for each material, emphasising the different sizes of the nanostructures: AgNWs are up to tens of micron long and very stiff; CNTs are shorter than one micron, with a flexible and easy to adapt shape, while PEDOT:PSS constitutes a uniform soft layer.
3.2. Optical Characterization. The transmittance over the visible spectrum $(350 \mathrm{~nm}$ to $750 \mathrm{~nm}$ ) are shown in Figure 6(a). Here, we see similar transmittance values for CNT and AgNWs, which become more transparent for longer wavelengths. PEDOT:PSS shows the lowest transmittance, and it is characterized by a strong absorption in the red part of the spectrum. As per previous protocols defined in literature, the transmittance value in the maximum sensitivity of the human eye in the green range $(550 \mathrm{~nm})$ of the spectrum is considered as reference transmittance, indicating transmittance values of $87 \%, 90 \%$, and $78 \%$ for AgNWs, CNTs, and PEDOT:PSS, respectively. Figure 6(b) illustrates the decrease in the transmittance with the increase in the number of spray-deposited layers. As the purpose of this work is to achieve semitransparent thermocouples, we have to look for a trade-off between sheet resistance and transmittance for each material. Both transmittance and sheet resistance vary according to the thickness. The thicker the layers are, the lower the sheet-resistance result and the lower the transmittances obtained. Therefore, in order to not lose transmittance, the number of layer used to fabricate the thermocouples was set to 10 .

3.3. Electrical Characterization. The resistivity of AgNW, PEDOT:PSS, and CNT deposited film is presented in Table 1 . Due to the very high resistivity of CNT compared to the other two films, the resistivity of a full TC is determined by the CNT, when this is part of it.

The measured sheet resistance of 10-layer single material thin films is also included in Table 1, showing how this parameter for PEDOT:PSS is two orders of magnitude lower than CNTs and one higher than AgNWs. Combining these numerical value, it is easy to estimate the FoM, equal to 45, 0.56, and 0.003 for AgNWs, PEDOT:PSS, and CNTs, respectively.

3.4. Thermoelectric Characterization. The measurement of the open-circuit voltage (Figure 7) gave a clearly linear relationship $\left(R^{2}>0.995\right)$ to temperature difference between hot and cold junction, for the temperature range used in the tests. As such, the Seebeck coefficient has been estimated as the slope of the curve, neglecting any second-order effect. The transparent TCs presented Seebeck coefficients of 50.4 $\pm 5.68 \mu \mathrm{V} / \mathrm{K}, \quad 26.9 \pm 2.87 \mu \mathrm{V} / \mathrm{K}$, and $14.8 \pm 0.83 \mu \mathrm{V} / \mathrm{K}$ for AgNWs-CNTs, CNTs-PEDOT, and AgNWs-PEDOT, respectively. These results are in line with previous literature for nontransparent nanomaterial-based thermocouples and, hence, represent a significant advancement towards the obtainment of transparent electronics. Nevertheless, not only the value is different among different material couples but also the same material couple, when sprayed in a different order results in different thermovoltages. This is particularly true for TC, in which one of the two materials is CNTs. For instance, when AgNWs are sprayed on top of CNTs, the Seebeck coefficient of the TC exhibits a drop of nearly $30 \%$, with respect to the reverse spraying order. An opposite to this phenomenon can be appreciated for the combination PEDOT:PSS and CNTs, in which the TCs with a CNT bottom layer outperform the dual 


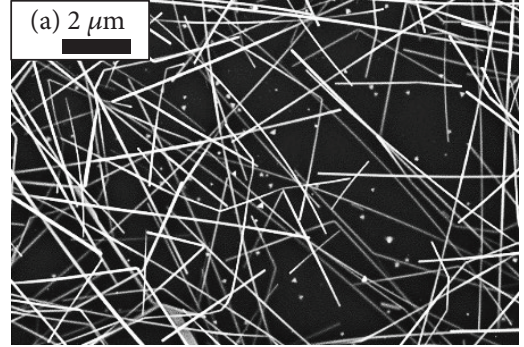

(a)

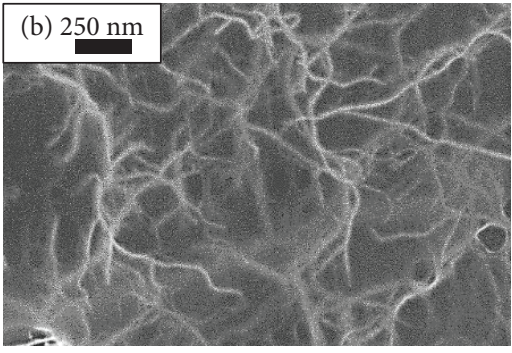

(b)

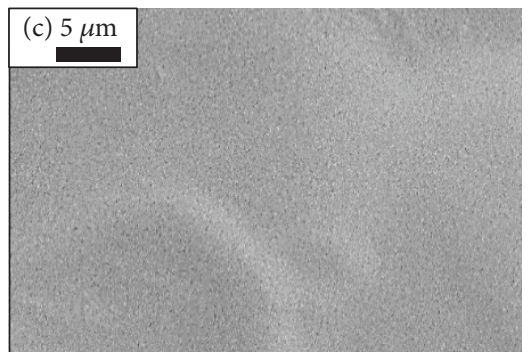

(c)

Figure 5: FESEM pictures of (a) AgNWs, (b) CNTs, and (c) PEDOT:PSS spray-deposited films.
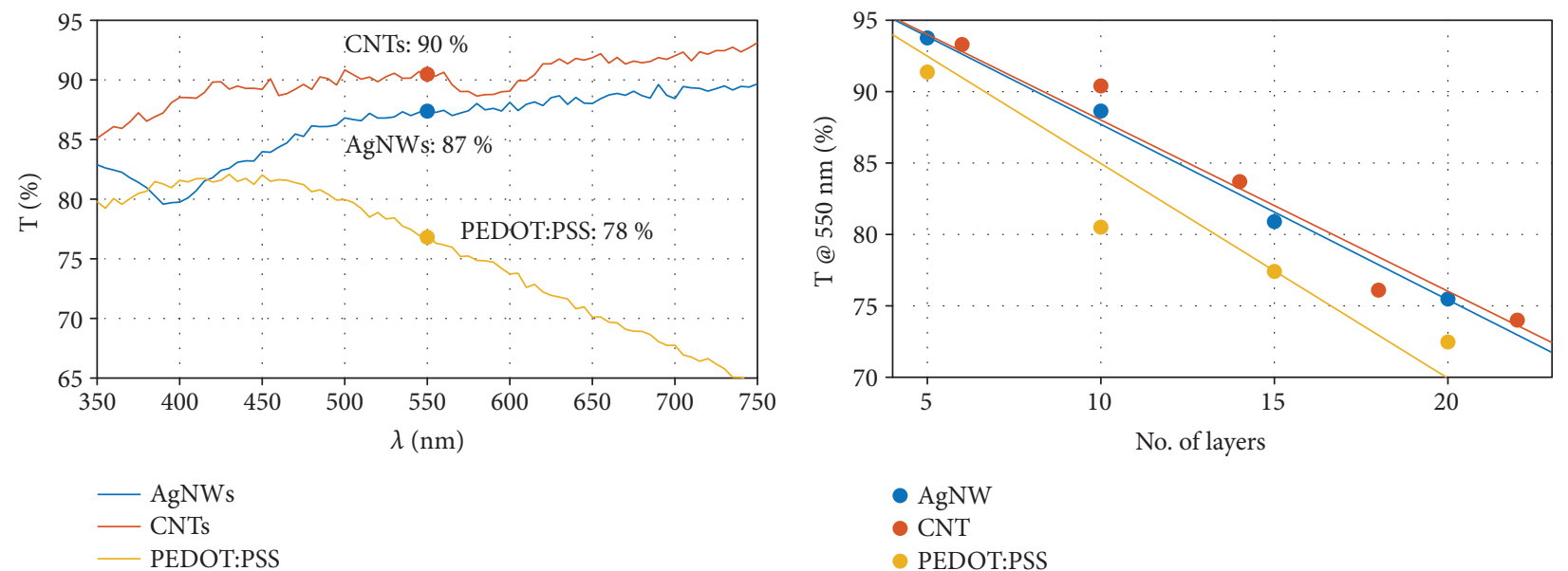

FIgure 6: Transmittance of reference films for the materials used. CNT and AgNW have similar transparency. PEDOT transparency decreases at increasing wavelengths.

TABLE 1: Electrical and optical properties of single material thinfilm.

\begin{tabular}{lccc}
\hline Material & $T(\%)$ at $550 \mathrm{~nm}$ & $R_{S}[\Omega / s q]$. & FoM (\%) \\
\hline AgNW & 85 & 52 & 45 \\
PEDOT:PSS & 76 & 100 & 0.650 \\
CNT & 89 & $3.4 \cdot 10^{4}$ & 0.003 \\
\hline
\end{tabular}

combination. The reasons behind the two behaviours are most likely related to a better electrochemical interface in the most performing combination, due to the sequence of process steps. In the former case, i.e., CNTs on AgNWs, the appropriate interface is guaranteed by the ductility and conformability of the CNTs, which can well entangle the AgNWs. Vice versa, the stiff AgNWs would not be able to conform to the rough surface of the CNTs, leading to poor electrical contact. In the latter case, i.e., PEDOT:PSS on CNTs, the motivation can be found in the solubility of PEDOT:PSS in water. After the deposition of CNTs on top of the polymeric layer, they need to be placed in a water bath to remove the dispersing matrix. By doing so, the CNTs become conductive, while part of the PEDOT:PSS is delaminated because of the contact with water. Finally, in the case of PEDOT:PSS and AgNW thermocouples, there is no difference in the deposition order, although this TC combination presents the lowest $V_{\text {OC }}$ (about $15.5 \mu \mathrm{V} / \mathrm{K}$ ). 


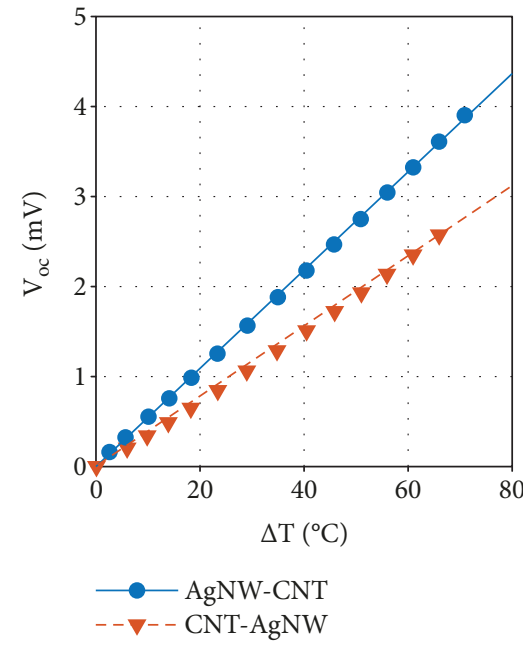

(a)

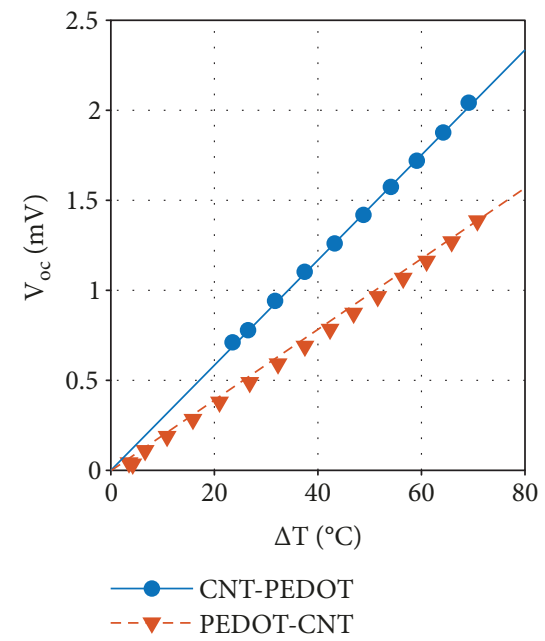

(b)

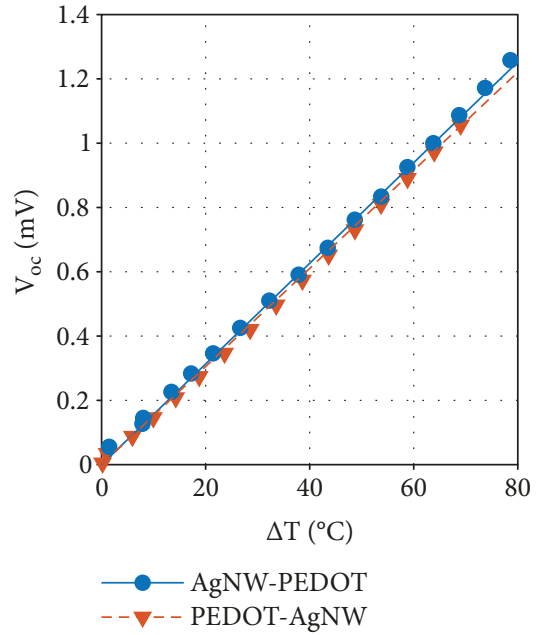

(c)

FIGURE 7: Linearity of open-circuit voltage over the temperature difference for thermocouple pairs made with (a) AgNW-CNTs, (b) CNTsPEDOT:PSS, and (c) AgNW-PEDOT:PSS. The order of mentioning in the legend indicates the spraying order with the second material sprayed after the first one.

TABLE 2: Series Seebeck coefficient of the four legs of the TCs in $\mu \mathrm{V} / \mathrm{K}$ for the three material combinations and the two spraying orders and the mean value of the individual junctions $\left(\bar{S}_{i}\right)$. The material mentioned first was sprayed first. The resistance and the resulting power over the four thermocouples are also displayed.

\begin{tabular}{lcccccc}
\hline Type & AgNW-CNT & CNT-AgNW & CNT-PEDOT:PSS & PEDOT:PSS-CNT & AgNW-PEDOT:PSS & PEDOT:PSS-AgNW \\
\hline $\bar{S}_{i}(\mu \mathrm{V} / \mathrm{K})$ & $50.4 \pm 5.68$ & $37.5 \pm 1.40$ & $26.9 \pm 2.87$ & $13.8 \pm 5.51$ & $14.8 \pm 0.83$ & $14.3 \pm 0.64$ \\
$S_{\text {series }}(\mu \mathrm{V} / \mathrm{K})$ & 201.7 & 149.9 & 107.4 & 55.1 & 58.9 & 57.2 \\
$R_{\text {series }}(\mathrm{k} \Omega)$ & 92.2 & 90.2 & 179 & 222 & 5.38 & 3.73 \\
$\mathrm{PF}_{\text {series }}\left(\mathrm{fW} / \mathrm{K}^{2}\right)$ & 110 & 62.3 & 16.1 & 3.42 & 161 & 219 \\
\hline
\end{tabular}

Table 2 presents the average voltage $\bar{S}_{i}$. The spraying order is indicated in each column, mentioning first the material deposited as bottom layer. We also analyzed the potential of these thermocouples as energy harvesters. For this purpose, we measured resistance values of the series of four thermocouples (also included in Table 2). PEDOT:PSS in combination with AgNWs shows lower resistance. Actually, the higher values obtained by the other TCs are associated to the high sheet-resistance value of the sprayed CNTs. The power factor was estimated as the square of the half of the Seebeck coefficient divided by the resistance (4). These values are included in Table 2. Errors have been calculated as the standard deviation.

$$
\mathrm{PF}_{\text {series }}=\frac{\left(S_{\text {series }} / 2\right)^{2}}{R_{\text {series }}}=\frac{1}{4} \frac{S_{\text {series }}^{2}}{R_{\text {series }}}
$$

It can be seen that the lower output power values are found in those thermocouples with CNTs. This very low values are caused by the high resistance of CNT layers (sheet resistance in the order of tens of $\mathrm{k} \Omega / \mathrm{sq}$ ). In contrast with this, the higher output power is achieved by the AgNWs-PEDOT:PSS combination where the sheet resistance is in all cases below $100 \Omega /$ sq.
Table 3 summarizes the Seebeck coefficients obtained in other planar thermocouples reported in literature. As the combination of materials is similar, the Seebeck coefficients do not vary significantly; they are, however, manufactured with high-throughput, large area methods. Albeit remarkable, this result, by itself, does not allow the employability of these printed TCs as energy harvesters because of the high leg-resistance.

In order to enhance the performance of the CNT-based thermocouples as thermal harvesters, a drastic reduction of the sheet resistance would be necessary. To achieve this, it would be needed to spray more layers with the consequential loss of transparency [36] or by using a third material to improve the junction [37].

\section{Conclusions}

We have demonstrated the feasibility of producing spraydeposited, large-scale, and semitransparent thermocouples on flexible substrates. These thermocouples show Seebeck coefficients of up to $54 \mu \mathrm{V} / \mathrm{K}$ and a very linear response, being a perfect candidate for autonomous temperature sensing. The best combination in terms of Seebeck coefficient was found for the junction made of AgNWs and CNTs, 
TABLE 3: Comparison among planar thermocouples.

\begin{tabular}{lccc}
\hline Reference & Thermocouple type & Seebeck coefficient $(\mu \mathrm{V} / \mathrm{K})$ & Temperature range $(\mathrm{K})$ \\
\hline Yu et al. [17] & Doped multiwalled CNT (MWCNT) bundles with metal & 22 & $110-300$ \\
Penza et al. [22] & contact [17] & 40.7 & $293-343$ \\
Miao et al. [38] & CNT bundles with Cr-Al electrodes & $\sim 35$ & $260-420$ \\
& Isolated MWCNTs with Pt-electrodes & 50.4 & $293-273$ \\
This work & AgNWs-SWCNTs & 26.9 & 14.8 \\
& SWCNTs-PEDOT:PSS & AgNWs-PEDOT:PSS & \\
\hline
\end{tabular}

depositing them in the mentioned order. The achievement of such semitransparent devices, easy to integrate into other systems with means of conventional solution-processing methods, is a first step towards transparent electronic devices, seamlessly integrated into windows or windshields. Finally, although further work is still necessary to overcome the current limitations, such as the high resistance, which hinders the use as energy harvesters, the methods we describe in this work, coupled with a choice of materials more tailored to the applications, can be generalised and employed for the realization of thermally sensitive electronics, where transparency, cost effectiveness, and ease of process are required.

\section{Data Availability}

The corresponding author is available to provide the interested reader with the raw data of the measurements for all the performed experiments.

\section{Conflicts of Interest}

The authors declare that they have no conflicts of interest.

\section{Acknowledgments}

This work was partially funded by the TUM Graduate School and ASK Industries Italy, through the project VASM.

\section{Supplementary Materials}

Figure S1: composition of two thermal images to compensate the two distinctly different thermal emission coefficients of foam glass (0.9) and aluminum (0.08) on the heater (top, bright) and cooler (bottom, dark). The value for the aluminum plates was experimentally evaluated and found to match the predicted range (0.08-0.09). The transition of the emission coefficients at the 12 edges of the aluminum plates was neglected. In reality, a smooth transition between the about $225^{\circ} \mathrm{C}$ hot heater, the about $25^{\circ} \mathrm{C}$ warm foam glass, and the about $5^{\circ} \mathrm{C}$ cold heater has to be assumed. (Supplementary Materials)

\section{References}

[1] A. Dey, O. P. Bajpai, A. K. Sikder, S. Chattopadhyay, and M. A. S. Khan, "Recent advances in CNT/graphene based thermoelectric polymer nanocomposite: a proficient move towards waste energy harvesting," Renewable and Sustainable Energy Reviews, vol. 53, pp. 653-671, 2016.

[2] S. Wang and D. D. L. Chung, "Carbon fiber polymer-matrix composite interfaces as thermocouple junctions," Composite Interfaces, vol. 6, no. 6, pp. 519-529, 1998.

[3] O. Yamashita, S. Tomiyoshi, and K. Makita, "Bismuth telluride compounds with high thermoelectric figures of merit," Journal of Applied Physics, vol. 93, no. 1, pp. 368-374, 2003.

[4] M. H. Elsheikh, D. A. Shnawah, M. F. M. Sabri et al., "A review on thermoelectric renewable energy: principle parameters that affect their performance," Renewable and Sustainable Energy Reviews, vol. 30, pp. 337-355, 2014.

[5] W. Honda, S. Harada, T. Arie, S. Akita, and K. Takei, "Wearable, human-interactive, health-monitoring, wireless devices fabricated by macroscale printing techniques," Advanced Functional Materials, vol. 24, no. 22, pp. 32993304, 2014.

[6] S. Kim, A. Traille, H. Lee et al., "Inkjet-printed sensors on paper substrate for agricultural applications," in 2013 European Microwave Conference, pp. 866-869, Nuremberg, Germany, October 2013.

[7] C. Perera, C. H. Liu, and S. Jayawardena, "The emerging internet of things marketplace from an industrial perspective: a survey," IEEE Transactions on Emerging Topics in Computing, vol. 3, no. 4, pp. 585-598, 2015.

[8] V. Sanchez-Romaguera, M. A. Ziai, D. Oyeka et al., "Towards inkjet-printed low cost passive UHF RFID skin mounted tattoo paper tags based on silver nanoparticle inks," Journal of Materials Chemistry C, vol. 1, no. 39, pp. 6395-6402, 2013.

[9] M. Irimia-Vladu, “"Green” electronics: biodegradable and biocompatible materials and devices for sustainable future," Chemical Society Reviews, vol. 43, no. 2, pp. 588-610, 2014.

[10] J. Gong, S. B. Darling, and F. You, "Perovskite photovoltaics: lifecycle assessment of energy and environmental impacts," Energy \&Environmental Science, vol. 8, no.7,pp. 1953-1968, 2015.

[11] T. Mustonen, K. Kordás, S. Saukko et al., "Inkjet printing of transparent and conductive patterns of single-walled carbon nanotubes and PEDOT-PSS composites," Physica Status Solidi (b), vol. 244, no. 11, pp. 4336-4340, 2007.

[12] A. Sandström, A. Asadpoordarvish, J. Enevold, and L. Edman, "Spraying light: ambient-air fabrication of large-area emissive devices on complex-shaped surfaces," Advanced Materials, vol. 26, no. 29, pp. 4975-4980, 2014.

[13] A. Abdellah, B. Fabel, P. Lugli, and G. Scarpa, "Spray deposition of organic semiconducting thin-films: towards the fabrication of arbitrary shaped organic electronic devices," Organic Electronics, vol. 11, no. 6, pp. 1031-1038, 2010. 
[14] A. Falco, M. Petrelli, E. Bezzeccheri, A. Abdelhalim, and P. Lugli, "Towards 3D-printed organic electronics: planarization and spray-deposition of functional layers onto 3Dprinted objects," Organic Electronics, vol. 39, pp. 340-347, 2016.

[15] M. Binda, D. Natali, A. Iacchetti, and M. Sampietro, "Integration of an organic photodetector onto a plastic optical fiber by means of spray coating technique," Advanced Materials, vol. 25, no. 31, pp. 4335-4339, 2013.

[16] C. Meng, C. Liu, and S. Fan, "A promising approach to enhanced thermoelectric properties using carbon nanotube networks," Advanced Materials, vol. 22, no. 4, pp. 535-539, 2010.

[17] C. Yu, Y. S. Kim, D. Kim, and J. C. Grunlan, "Thermoelectric behavior of segregated-network polymer nanocomposites," Nano Letters, vol. 8, no. 12, pp. 4428-4432, 2008.

[18] M. Bobinger, D. Angeli, S. Colasanti, P. La Torraca, L. Larcher, and P. Lugli, "Infrared, transient thermal, and electrical properties of silver nanowire thin films for transparent heaters and energy-efficient coatings," Physica Status Solidi (a), vol. 214, no. 1, 2017.

[19] W. Hong, Y. Xu, G. Lu, C. Li, and G. Shi, "Transparent graphene/PEDOT-PSS composite films as counter electrodes of dye-sensitized solar cells," Electrochemistry Communications, vol. 10, no. 10, pp. 1555-1558, 2008.

[20] Z. Wu, Z. Chen, X. Du et al., "Transparent, conductive carbon nanotube films," Science, vol. 305, no. 5688, pp. 1273-1276, 2004.

[21] O. Bubnova, Z. U. Khan, A. Malti et al., "Optimization of the thermoelectric figure of merit in the conducting polymer poly (3, 4-ethylenedioxythiophene)," Nature Materials, vol. 10, no. 6, pp. 429-433, 2011.

[22] M. Penza, R. Rossi, M. Alvisi et al., "Thermoelectric properties of carbon nanotubes layers," in Sensors and Microsystems, pp. 73-79, Springer, 2011.

[23] A. Falco, L. Cinà, G. Scarpa, P. Lugli, and A. Abdellah, "Fully-sprayed and flexible organic photodiodes with transparent carbon nanotube electrodes," ACS Applied Materials \& Interfaces, vol. 6, no. 13, pp. 10593-10601, 2014.

[24] M. Schmidt, A. Falco, M. Loch, P. Lugli, and G. Scarpa, "Spray coated indium-tin-oxide-free organic photodiodes with PEDOT: PSS anodes," AIP Advances, vol. 4, no. 10, article 107132, 2014.

[25] J. Krantz, T. Stubhan, M. Richter et al., "Spray-coated silver nanowires as top electrode layer in semitransparent P3HT: PCBM-based organic solar cell devices," Advanced Functional Materials, vol. 23, no. 13, pp. 1711-1717, 2013.

[26] K. Zilberberg, F. Gasse, R. Pagui et al., "Highly robust indium-free transparent conductive electrodes based on composites of silver nanowires and conductive metal oxides," Advanced Functional Materials, vol. 24, no. 12, pp. 16711678, 2014.

[27] M. Bobinger, V. Dergianlis, A. Albrecht et al., "Solution processing of silver nanowires for transparent heaters and flexible electronics," in 2017 13th Conference on Ph.D. Research in Microelectronics and Electronics (PRIME), pp. 9-12, Giardini Naxos, Italy, June 2017.

[28] J. H. Lee, K. Y. Lee, M. K. Gupta et al., "Highly stretchable piezoelectric-pyroelectric hybrid nanogenerator," Advanced Materials, vol. 26, no. 5, pp. 765-769, 2014.
[29] T. Park, J. Na, B. Kim, Y. Kim, H. Shin, and E. Kim, "Photothermally activated pyroelectric polymer films for harvesting of solar heat with a hybrid energy cell structure," ACS Nano, vol. 9, no. 12, pp. 11830-11839, 2015.

[30] J. J. Lee, D. Yoo, C. Park, H. H. Choi, and J. H. Kim, “All organic-based solar cell and thermoelectric generator hybrid device system using highly conductive PEDOT: PSS film as organic thermoelectric generator," Solar Energy, vol. 134, pp. 479-483, 2016.

[31] W. Cao, J. Li, H. Chen, and J. Xue, "Transparent electrodes for organic optoelectronic devices: a review," Journal of Photonics for Energy, vol. 4, no. 1, article 040990, 2014.

[32] A. Abdellah, A. Abdelhalim, F. Loghin et al., "Flexible carbon nanotube based gas sensors fabricated by large-scale spray deposition," IEEE Sensors Journal, vol. 13, no. 10, pp. 40144021, 2013.

[33] F. Loghin, S. Colasanti, A. Weise et al., "Scalable spray deposition process for highly uniform and reproducible CNT-TFTs," Flexible and Printed Electronics, vol. 1, no. 4, article 045002, 2016.

[34] G. Haacke, "New figure of merit for transparent conductors," Journal of Applied Physics, vol. 47, no. 9, pp. 4086-4089, 1976.

[35] A. Falco, P. Lugli, F.-C. Loghin, and A. Rivadeneyra, "Design, simulation and fabrication strategies for printed out-of-plane thermoelectric devices," in 2017 IEEE Sensors, pp. 1-3, Glasgow, UK, November 2017.

[36] A. Falco, J. Salmerón, F. Loghin, A. Abdelhalim, P. Lugli, and A. Rivadeneyra, "Optimization of process parameters for inkjet printing of CNT random networks on flexible substrates," in 2016 IEEE 16th International Conference on Nanotechnology (IEEE-NANO), pp. 487-490, Sendai, Japan, August 2016.

[37] A. Dey, A. Maity, M. A. S. Khan, A. K. Sikder, and S. Chattopadhyay, "PVAc/PEDOT: PSS/graphene-iron oxide nanocomposite (GINC): an efficient thermoelectric material," RSC Advances, vol. 6, no. 27, pp. 22453-22460, 2016.

[38] T. Miao, S. Shi, S. Yan et al., "Integrative characterization of the thermoelectric performance of an individual multiwalled carbon nanotube," Journal of Applied Physics, vol. 120, no. 12, article 124302, 2016. 


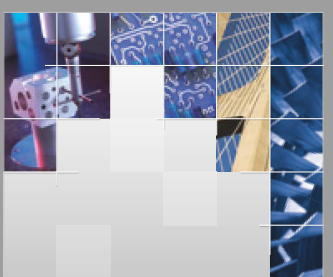

\section{Enfincering}
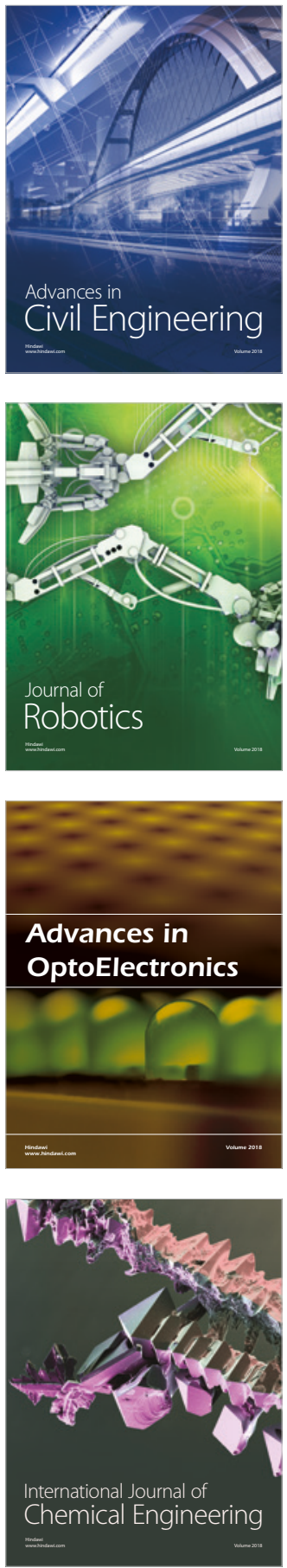

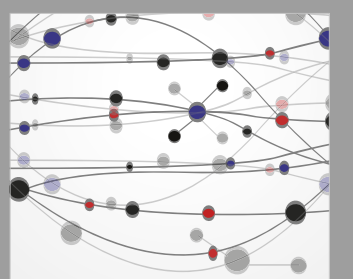

\section{Rotating \\ Machinery}

The Scientific World Journal

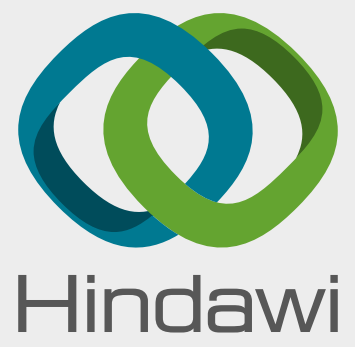

Submit your manuscripts at

www.hindawi.com
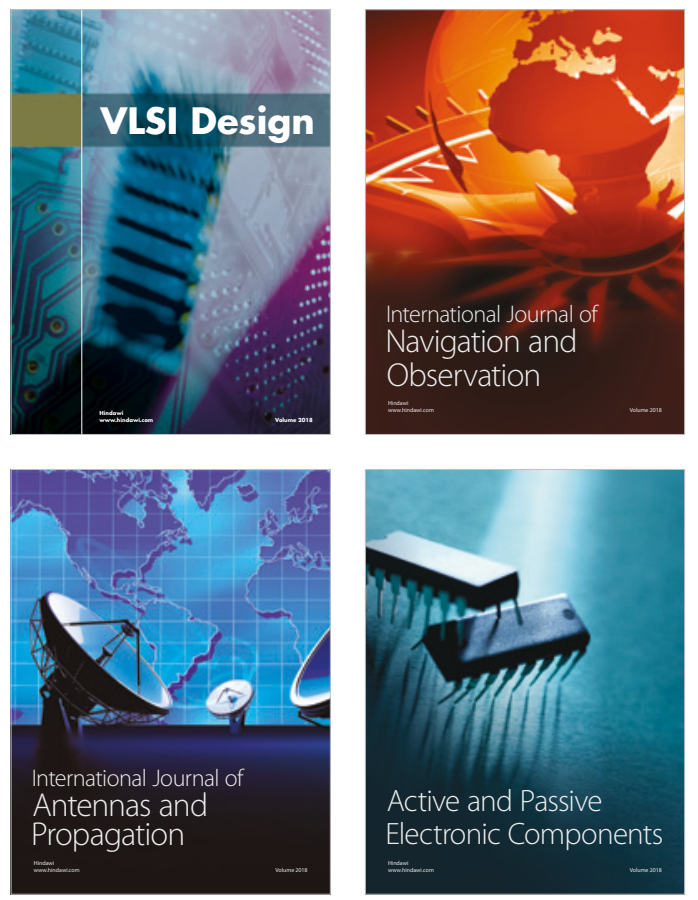
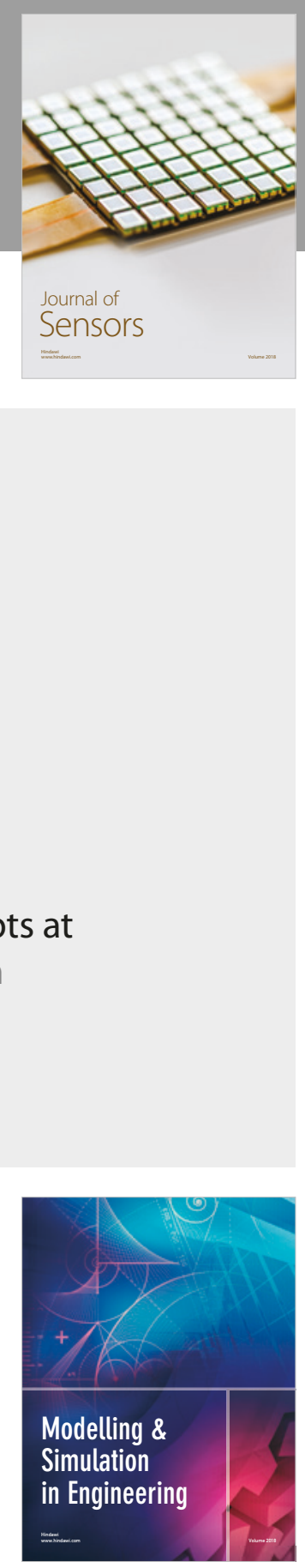

\section{Advances \\ Multimedia}
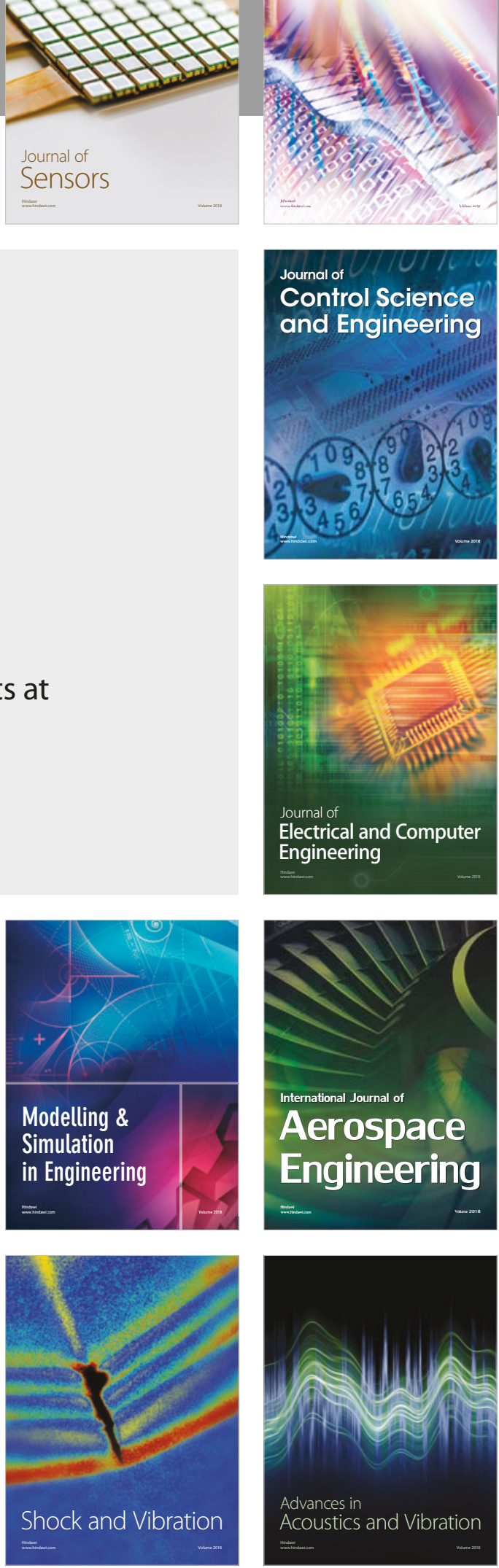